2. (v) antitative Bestimmung organischer Köper.

\title{
a. Elcmentaranalyse.
}

Einige Abänderungen in der vereinfachten Elementaranalyse beschreibt M. Dennstedt $t^{1}$ ). Die wichtigste Änderung ist die Vereinfachung dar doppelten Sauerstoffzufübrung, deren Einrichtung aus der nachstehenden Abbildung (Fig. 37) ersichtlich ist. Die Quetschhähne sind durch Glashähne ersetzt und der durch den Blasenzähler gehende Sanerstoff wird schon vorher mit Chlorkalzium getrocknet. Die feinste

Fig. 37.

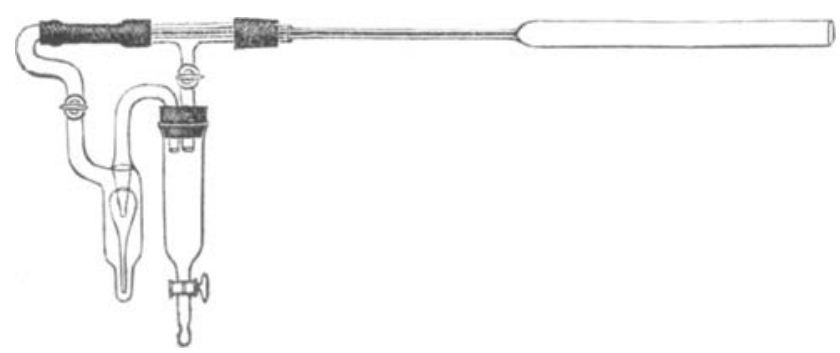

Regulierung des Sauerstoffstromes wird dadurch ermöglicht, dass man. die Durchbohrung der Küken an den Glashähnen mit einer scharfenKantenfeile in der Drehrichtung nach beiden Seiten spitz zulaufend einfeilt. Das Füllen des Blasenzählers geschieht mit einer fein ausgezogenen Pipette durch die U-förmige Krümmung des umgekehrt gehaltenen Blasenzählers.

Das zuweilen vorkommende Aufwärtsbiegen des Verbrennungsrohres in der Mitte wird dadurch verhindert, dass man einen etwa 4 cn langen,.. mit Asbest gefütterten, $\mathbf{U}$-förmig gebogenen Eisenblechstreifen dicht hinter der Kontaktmasse über das Rohr hängt. Die beiden Enden des Blechstreifens sind durch Bleigewichte ausreichend beschwert.

In die Einschnürung des Trockenturms für den Sanerstoff wird zum Schutze des Natronkalks gegen etwaiges Spritzen der Schwefelsäure bei lebhaftem Gasstrom ein kleiner Trichter mit etwas seitwärts. gebogenem Rohr eingesetzt. In den Trichter legt man etwas Glaswolle.

i) Chemiker-Zeitung' 33, 769 . 
Zur Verstärkung der katalytischen Wirkung des Platins wird bekanntlich an die vordere Öse des in das Imnenrohr eiuzulegenden Glasstabes ein Bündel feinsten Platindrahtes gebunden. Nach Meerwei In wird dieser Glasstab statt dessen mehrfach mit dünnem Platindraht umwunden. Diesen Platindraht sieht man dann wälrend der Verbrennung mehr oder weniger tief in das Innenrohr hinein aufglähen. was einen sehr guten Anhalt für den Verlauf der Verbrennung gibt. Ausserdem wird durch den Draht ein Anschmelzen des Glasstabes an das Inneurohr verhindert.

Das tragbare Universalstativ wird jetzt ron der Firma Dittmar und Vierth in Hamburg auch mit kurzen Füssen geliefert, so dass es auf jedem beliebigen Tisch aufgestellt werden kann.

Dem Azotometer für die Stickstoffbestimmung nach Fig. 38. Dumas ist die in der Abbildung Fig. 38 dargestellte ver-

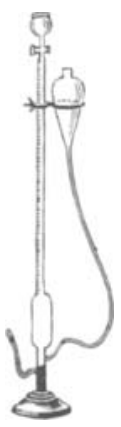
änderte Form gegeben, die hinreichenden Fassungsraum mit der Möglichkeit genauen Ablesens verbindet, indem das Rohr dicht über dem Ansatzrohr erweitert und in seinem oberen Teil ziemlich eng gehalten ist.

Dennstedt und Hassler ${ }^{1}$ ) haben vor einiger Zeit darauf aufmerksam gemacht, dass das zur Verwendung kommende Bleisuperoxyd mennigehaltig sein und besonderen Ansprüchen an Reinheit genügen muss. Da ein derartiges Produkt im Handel nicht erhältlich ist, muss man es sich selbst darstellen, Dies geschieht, indem man eine möglichst grosse Menge des im Handel unter der Bezeichnung $\gg \mathrm{nach}$ Dennstedt $z$ zu beziehenden Bleisuperoxyds im Verbrennungsrohr zur Umwandlung in Mennige auf $400-450^{\circ} \mathrm{C}$. erhitzt, während man Luft darüber leitet. Diese Mennige mischt man mit der gleichen Menge unveränderten Bleisuperoxyds, welches für sich zur Abspaltung etwa vorhandener Kohlensäure auf $320-350^{\circ} \mathrm{C}$. erhitzt worden ist.

\section{b. Bestimmung näherer Bestandteile.}

Eine neue Methode zur raschen Bestimmung von Alkohol teilt D. Sidersky ${ }^{2}$ ) mit. Diese von H. Rapeller vorgeschlagene Methode beruht auf der Mischbarkeit von Äther mit starkem Alkohol. Man

1) Chemiker-Zeitung 33, 133; vergl. diese Zeitschrift 50, 309.

2) Bull. assoc. chim. sucr. et dist. 27, 562; durch Journ, of the soc. of shem. industry 29, 104; vergl. hierzu diese Zeitschrift 47,68. 